\title{
Finite-State Markov Fading Channel Based Dynamic Spectrum Access in Cognitive Radio Networks
}

\author{
Yebin Tao1 \\ Bengbu Automobile NCO Academy, \\ Anhui, China \\ e-mail: yebintao@163.com \\ Zhu Shiding2 \\ Bengbu Automobile NCO Academy, \\ Anhui, China \\ e-mail: zhushiding@163.com
}

\author{
Lingfang Zou3 \\ 73122 Army \\ Fujian, China \\ e-mail: zoulingfang@163.com \\ Junye Meng4 \\ 73122 Army \\ Fujian, China \\ e-mail: junyemeng@163.com
}

\begin{abstract}
In this paper, we propose a finite-state markov fading channel (FMFC) model to investigate the dynamic spectrum access in fading channel cognitive radio networks. Most existing work failed to take the channel fading into consideration. Based on the important characteristic of fading for wireless channel, we consider both the idle probability of the primary user and the capacity of channel which is related to the channel fading. To obtain the better performance and acceptable complexity, we design an expected channel capacity based dynamic spectrum access strategy. Simulation results verify the improvement on throughput of the proposed approach.
\end{abstract}

Keywords: cognitive radio; dynamic spectrum access; FMFC; Markov; channel selection

\section{INTRODUCTION}

Dynamic Spectrum Access (DSA) [1] has drawn great attention in recent years, since it is the promising solution to the spectrum shortage. In the cognitive radio networks (CRNs) [2], the secondary users (SUs) which have the ability to carry out spectrum sensing and dynamic spectrum accessing channel could use the idle channel which is not occupied by the primary user (PU). How to design an efficient approach for DSA is an important problem in the CRN.

Recent years, many researches paid much attention to the strategies based on the partially observable markov model (POMDP) to improve the performance of DSA. A decentralized MAC protocol based on the POMDP framework was investigated to maximize the expected throughput in [3]. The cost and the throughput gain were analyzed in [4]. In [5], a multi-channel MAC protocol based on cooperative spectrum sensing was designed. The authors analyzed the optimality and complexity, and the impacts of sensing errors in [6] and in [7] respectively. The condition where the un-slotted time frame was assumed was analyzed in [8]. Also, the channel selection problem in multi users scenario was also studied by using the game theory [9]-[11]. The channel sequentially exploring methods by using the optimal stopping approach for OSA systems were also studied in [12]-[13], where the expected performance and the cost paid were both taken into consideration, and a rational action that stopping or proceeding to observe would be made by considering the balance. The Multi-armed bandit was used in the OSA problem in [14]-[15], where a user collect the statistics of the candidate channels and then chooses one or more channels among several candidate channels when the statistics information are unknown.

However, one of the most important characteristic of wireless channels is the fading, which would bring important effect on the channel capacity. Most existing work failed to consider the channel fading in their DSA model and the access approach, which limits their application in practical scenarios.

To design an efficient DSA approach in the practical scenarios, towording the fading channel character in wireless networks, we propose a finite-state markov fading channel (FMFC) model, and design the greedy algorithm based on the POMDP, considering both the idle probability of the primary user and the capacity of channel which is related to the channel fading.

The remainder of the paper is organized as follows. FMFC model and the greed channel choosing algorithm is proposed in Section II. The simulation results and the discussion are presented in section III. We draw the conclusion in section IV.

\section{THE FMFC MODEL AND GREED ALGORITHM}

The scenario we considered is a time-varying CRN consisted by $N$ fading PU channels. Define 
$\boldsymbol{P}(t)=\left[P_{1}(t), P_{2}(t), \cdots, P_{N}(t)\right]$ as the PU channel state, where $P_{i}(t)$ is the state of the $i$-th PU channel:

$$
P_{i}(t)=\left\{\begin{array}{l}
1, \text { busy } \\
0, \text { idle }
\end{array}\right.
$$

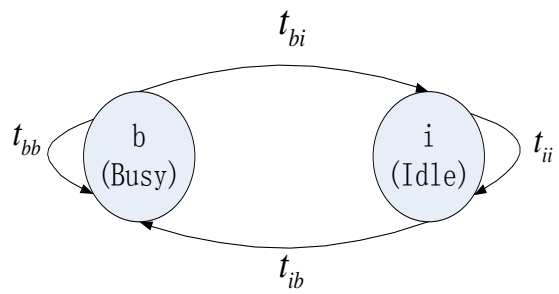

Figure 1. The state transition of PU channel.

The $M=2^{N}$ states network is depicted as a finite-state Markov fading channel (FMFC) model, the state transition probability matrix is get as follows:

$$
\boldsymbol{T}=\left[\begin{array}{cccccc}
t_{00} & t_{01} & 0 & 0 & \cdots & 0 \\
t_{10} & t_{11} & t_{12} & 0 & \cdots & 0 \\
0 & t_{21} & t_{22} & t_{23} & \cdots & 0 \\
\vdots & \vdots & \vdots & \vdots & \vdots & \vdots \\
0 & 0 & \cdots & t_{K-3, K-2} & t_{K-2, K-2} & t_{K-2, K-1} \\
0 & 0 & \cdots & 0 & t_{K-1, K-2} & t_{K-1, K-1}
\end{array}\right],
$$

where $t_{k, j}$ is given by the adjacent transfer method, calculated as:

$$
\left\{\begin{array}{l}
t_{k, k+1}=\sigma \frac{\sqrt{\frac{2 \pi_{k} \Lambda_{k}}{\gamma_{0}}} f_{d} e^{-\Lambda_{k} / \gamma_{0}}}{\int_{\Lambda_{k}}^{\Lambda_{k+1}} \chi(\gamma) d \gamma=e^{-\Lambda_{k} / \gamma_{0}}-e^{-\Lambda_{k+1} / \gamma_{0}}}, \\
k=0,1, \cdots, K-2 ; \\
u_{k, k-1}=\sigma \frac{\sqrt{\frac{2 \pi_{k} \Lambda_{k}}{\gamma_{0}}} f_{d} e^{-\Lambda_{k} / \gamma_{0}}}{\int_{\Lambda_{k}}^{\Lambda_{k+1}} \chi(\gamma) d \gamma=e^{-\Lambda_{k} / \gamma_{0}}-e^{-\Lambda_{k+1} / \gamma_{0}}}, \\
k=1,2, \cdots, K-1 ;
\end{array}\right.
$$

In equation ( 3$)$,

$\Lambda_{k}=e^{k \eta / B}-1, k \in\{0,1, \cdots, K-1\}$, and $\eta$ is the rate increment between adjacent channel quality states. $\sigma$ is packet duration time. $\gamma$ is the instantaneous signal-to-noise ratio, when $\Lambda_{k} \leq \gamma<\Lambda_{k+1}$, the data rate on this fading channel would be:

$$
\lambda_{k}=B \ln \left(1+\Lambda_{k}\right)
$$

We adopt typical multipath propagation for the fading channel, and the $\gamma$ would be exponential distribution, i.e., the probability density function is as follow:

$$
\chi(\gamma)=\frac{1}{\gamma_{0}} e^{-\gamma / \gamma_{0}}
$$

where $\gamma_{0}$ is the average value of signal-to-noise ratio.

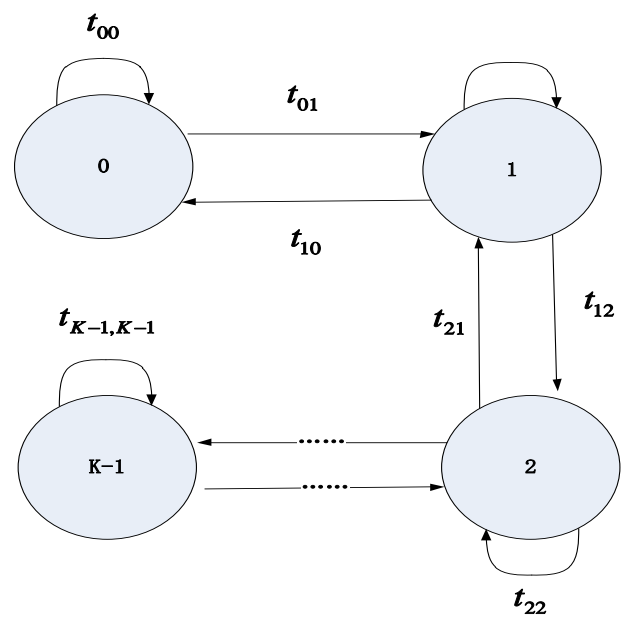

Figure 2. The FMFC model.

The proposed greedy algorithm is based on POMDP [3], because the SUs can usually only observe a part of the system state limited by the hardware capability and energy constrain. Although the quality of channel would not be observed when the channel is busy, it is still existing and changing over time in fact. We define the channel state information as $\square=\left\{q_{b}, q_{i 0}, q_{i 1}, \ldots q_{i, K-1}\right\}$, where $q_{b}$ denotes the channel is occupied by PU and the quality state cannot be observed, while $q_{i, k}$ denotes that the channel is idle and the capacity state is $k$. The transition probability is given by:

$$
\begin{aligned}
& \operatorname{Pr}\left\{\square{ }_{n}^{j+1}=q_{s_{2}, l} \mid \square{ }_{n}^{j}=q_{s_{1}, k}\right\}=t_{s_{1} k, s_{2} l} \\
& \left(s_{1}, s_{2}\right) \in\{b, i\},(k, l) \in\{0,1, \cdots K-1\}
\end{aligned},
$$

Where

$$
t_{s_{1} k, s_{2} l}= \begin{cases}q_{b b} u_{k, k+1} & s_{1}=s_{2}=b \\ q_{b i} u_{k, k+1} & s_{1}=b, s_{2}=i \\ q_{i i} u_{k, k+1} & s_{1}=s_{2}=i \\ q_{i b} u_{k, k+1} & s_{1}=i, s_{2}=b\end{cases}
$$

This following matrix (7) shows the transition probabilities that the channel state changes from one to another.

The SUs do the following two actions at the beginning of slot: determining a channel to sense and then making the decision whether to access this channel according to the sensing result. Denote the action as $m(t)$, and 


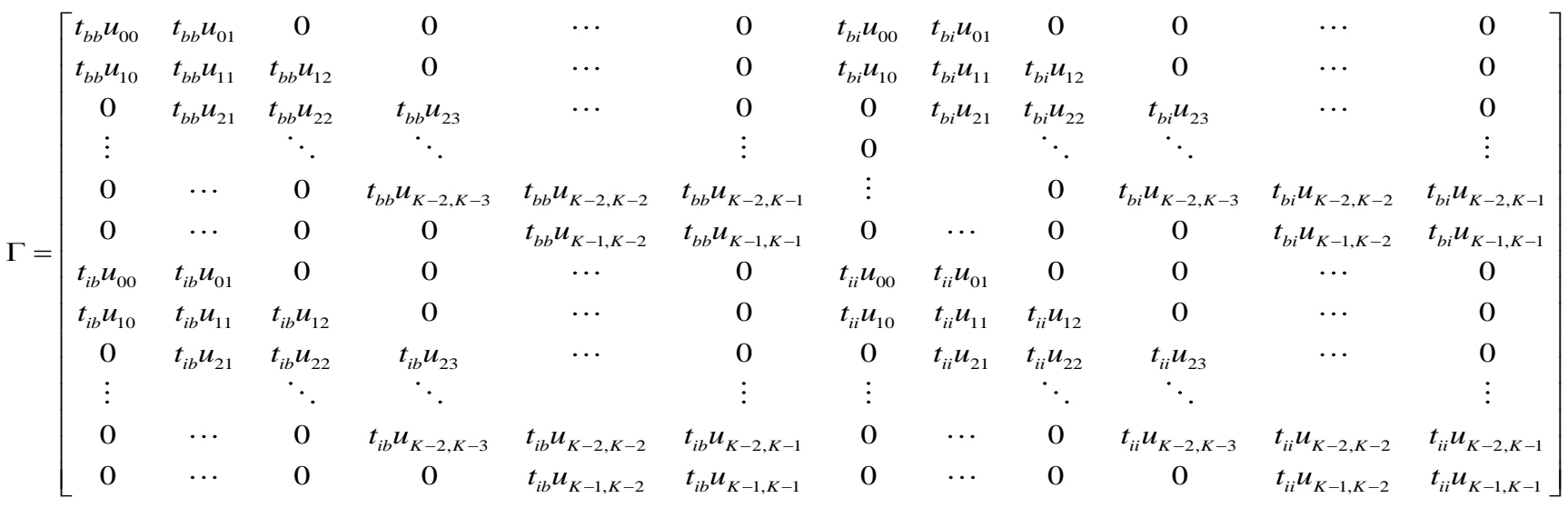

denote $\mathfrak{R}_{t}\left(\Omega(t), v_{i}(t)\right)$ as the received reward after implementing the action, where $\mathfrak{R}(t), v_{i}(t)$ are the belief vector and the quality vector respectively. In the proposed greedy algorithm, in order to reduce the complexity, we focuses the immediate reward. When channel quality state is $k$, the data rate $\lambda_{k}=B \ln \left(1+\Lambda_{k}\right)$. If the SU select channel $a$ in slot $t$, it will obtain the expected reward as follows:

$$
\begin{aligned}
& \Theta_{a}(t)=\sum_{k=0}^{K-1} \ell_{a}(t) \beta_{a}^{k} \lambda_{k} \sigma \\
& =\sum_{k=0}^{K-1} \ell_{a}(t) \beta_{a}^{k} B \ln \left(1+\Lambda_{k}\right) \sigma
\end{aligned}
$$

where $\ell_{a}(t)$ is the conditional probability that channel is idle and $\beta_{a}^{k}(t)$ is its quality state is $k$. The optimal channel $m^{*}(t)$ is selected to maximize the expected immediate reward:

$$
m^{*}(t)=\arg \max _{i=1,2, \cdots, N} \Theta_{i}(t)
$$

In order to maximize the total expected reward:

$$
\mathfrak{J}^{*}=\underset{\mathfrak{J}=[\xi(1), \cdots, \xi(T)]}{\arg \max } \mathrm{E}_{\mathfrak{I}}\left[\sum_{t=1}^{T} \Theta_{\varphi(t)}(t) \mid \mathfrak{R}(1), v(1)\right],
$$

We make the channel choosing decision according to the strategy policies:

$$
\mathfrak{I}=[\xi(1), \xi(2), \cdots, \xi(t), \cdots, \xi(T)]
$$

where $\varphi^{*}(t)$ is given by:

$$
\xi^{*}(t)=\arg \max _{\xi(t)} \mathrm{E}_{\xi(t)}\left[\Theta_{\xi(t)}(t) \mid \mathfrak{R}(t), v(t)\right]
$$

$$
t=1,2, \cdots, T
$$

\section{NUMERICAL RESULTS AND DISCUSSIONS}

We verify the proposed approach by the MATLAB simulation. Without the generality, the system is set up as follows: the number of channels is 5 , and the independent

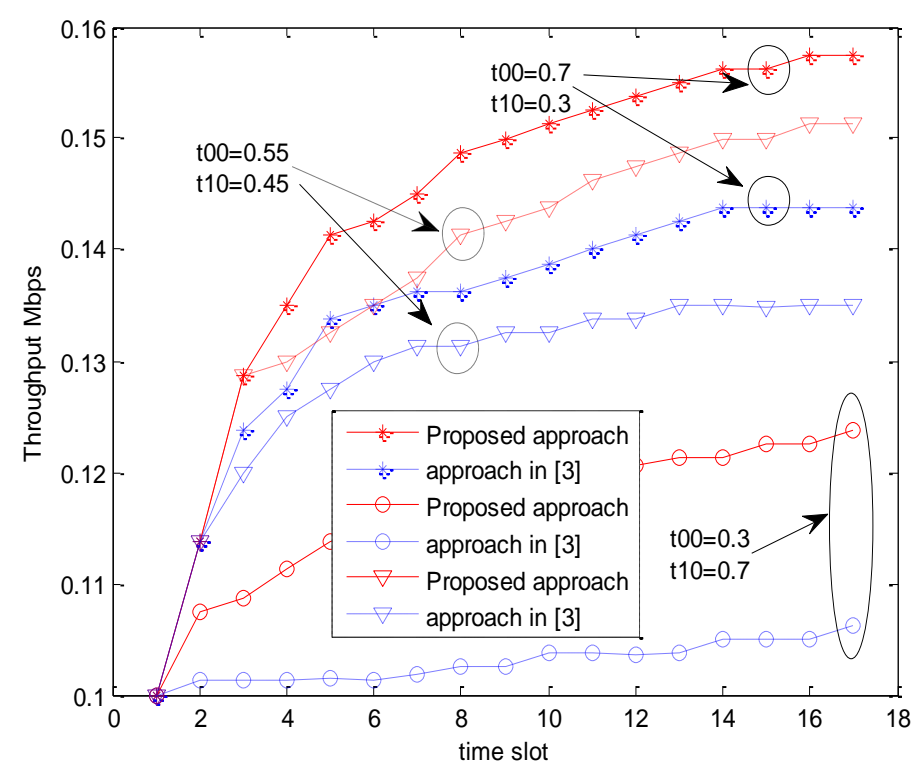

Figure 3. The proposed approach vs the idle-probabilityoriented approach in [3].

Markov states is 8 . The channel bandwidth is $1 \mathrm{MHz}$, the average SNR is $10 \mathrm{~dB}$, the doppler frequency is 0.033 , and the packet duration time is $\sigma=10 \mathrm{~ms}$. We show the simulation result when three different parameters are set respectively, i.e., we set the $t_{00}=0.55, t_{10}=0.45$;

$t_{00}=0.7, t_{10}=0.3 ; t_{00}=0.3, t_{10}=0.7$ to simulate three typical scenarios.

The simulation results are shown in Fig. 3. It can be seen in Fig. 3 that the proposed approach outperforms the idle-probability oriented approach in [3], which is the typical method in existing work. In the idle-probability oriented approach, the channel choosing decision is depend on the information of channel idle probablity in the next slots, the channel capacity variation caused by the fading was neglected. While in the proposed 
approach, we consider both the idle probability and the channnel capacity at the same time to obtain better performance.

\section{CONCLUSIONS}

This paper formulated a finite-state markov fading channel (FMFC) model for the dynamic spectrum access decision in fading channel environment. In most existing work, the channel fading, which is an important characteristic of wireless channel, was not taken into consideration in the process of channel selection process based on the traditional POMPD approach. The main motivation was that the channel choosing should take the channel fading into consideration in the wireless networks. In the proposed channel choose approach, we considered both the channel idle probability and the achievable data rate on the channel which is determined by the fading. Simulation results showed that the proposed approach could achieve better performance compared with the traditional POMPD based idle-oriented channel selection approach in existing work.

\section{REFERENCES}

[1] Zhang Honggang, Zhou Xiaofei, Yazdandoost. K. "Multiple Signal Waveforms Adaptation in Cognitive Ultra-Wideband Radio Evolution," IEEE Journal on selected areas in communications, , vol. 24, no. 4, Apr. 2006.

[2] J. Mitola and G. Q. Maguire, "Cognitive radios: making software radios more personal," IEEE Personal Communications, vol. 6, no. 4, 2003.

[3] Zhao Qing, Tong Lang, Swami. A, "Decentralized Cognitive MAC for Opportunistic Spectrum Access in Ad Hoc Networks: A POMDP Framework," IEEE Selected Areas in Commun., vol. 25, no. 3, Apr. 2007

[4] Hoang. A, Liang Yingchang, Wong. D, "Opportunistic spectrum access for energy-constrained cognitive radio," IEEE 67th Vehicular Technology Conference-Spring, vol. 1-7, pp. 1559-1563, 2008.
[5] Ahmad. S, Liu. Mingyan, Javidi. T, "Optimality of Myopic Sensing in Multichannel Opportunistic Access," IEEE Transactions on Information Theory, vol. 55, pp. 4040-4050, Sep. 2009.

[6] Chen Yunxia, Zhao Qing, Swami. A, "Bursty traffic in energy-constrained opportunistic spectrum access," IEEE Global Telecommunications Conference, vol. 1-11, pp. 4641-4646, 2007.

[7] Chen Yunxia, Zhao Qing, Swami. A, "Joint design and separation principle for opportunistic spectrum access in the presence of sensing errors," IEEE Transactions on Information Theory, vol. 54, pp. 2053-2071, May. 2008.

[8] Zhao Qing, Krishnamachari. B, and K. Q. Liu, "On Myopic Sensing for Multi-Channel Opportunistic Access: Structure, Optimality, and Performance," IEEE Transactions on Wireless Communications, vol. 7, pp. 5431-5440, Dec. 2008.

[9] ANANDKUMAR A, MICHAEL N, TANG Ao. Opportunistic spectrum access with multiple users: Learning under competition [C]. Proc. IEEE Infocom, March 2010, San Deigo, USA.

[10] XU Yuhua, ANPALAGAN A, WU Qihui, et al. Decision-Theoretic Distributed Channel Selection for Opportunistic Spectrum Access: Strategies. Challenges and Solutions [J]. IEEE communications survey \& tutorial. To appear.

[11] XU Yuhua, WU Qihui, WANG Jinlong, Game theoretic channel selection for opportunistic spectrum access with unknown prior information [C]. In Proc. IEEE ICC, 2011.

[12] SABHARWAL A, KHOSHNEVIS A, KNIGHTLY E Opportunistic spectral usage: bounds and a multi-band CSMA/CA protocol [J]. IEEE/ACM Trans. Network., 2007, vol. 15 , no. 3 , pp. 533-545.

[13] CHENG Hoting, ZHUANG Weihua. Simple channel sensing order in cognitive radio networks [J]. IEEE J. Sel. Areas Commun. 2011, vol. 29, no. 4, pp. 676-688.

[14] MAHAJAN A, TENEKETZIS D. Multi-Armed Bandit Problems [C]. Foundations and Applications of Sensor Management, 2008, Springer US.

[15] FILIPPI S, CAPPE O, GARIVIER A. Optimally sensing a single channel without prior information: The tiling algorithm and regret bounds [J]. IEEE J. Sel. Topics Signal Process., Feb 2011, vol. 5 , no. 1 , pp.68-76. 